\title{
The 4-8 GHz Stochastic Cooling Upgrade for the Fermilab Debuncher*
}

\author{
D. McGinnis", FNAL, Batavia, IL
}

\begin{abstract}
During the Fermilab Collider Run II, the Main Injector is expected to provided a three-fold increase in flux of the proton beam sent to the antiproton production target as compared to the previous Collider Run Ib. This increase in antiproton production rate to $20 \times 10^{10}$ antiprotons/hour will be handled by upgrading the stochastic cooling systems in both the Debuncher and Accumulator rings of the Fermilab Antiproton Source. In the Debuncher ring, the upgrade calls for increasing the stochastic cooling system bandwidth from 2-4 to 4-8 GHz. The effective front-end noise temperature of the cooling systems will also be lowered from $125 \mathrm{~K}$ to $35 \mathrm{~K}$. This paper will discuss some details of the system design of the new 4-8 $\mathrm{GHz}$ cooling systems in the Debuncher Ring.
\end{abstract}

\section{INTRODUCTION}

The original horizontal, vertical, and momentum Debuncher cooling systems covered a bandwidth of 2-4 GHz. The pickups and kickers each consisted of 128 $100 \Omega$ stripline pickups with an octave bandwidth. The signals were combined (or split) with a binary combiner tree. The pickups were cooled to $77 \mathrm{~K}$ and had an overall front-end system temperature of $125 \mathrm{~K}$. The kickers were powered with 1600 Watts per plane. These systems were power limited and operated well below optimum cooling gain. These systems could cool $45 \times 10^{6} 8 \mathrm{GeV}$ particles from $25 \pi$-mm-mrad (95\% un-normalized) to $5 \pi$-mmmrad in 2.4 seconds.

The original upgrade plan called for the same cooling bandwidth of $2-4 \mathrm{GHz}$ but with a reduction in noise temperature of the front-end to $35 \mathrm{~K}$. This was to be accomplished by cooling the pickup arrays and amplifiers to $10 \mathrm{~K}$ with liquid helium as a coolant. Also the plan called for the arrays to plunge (change the transverse spacing of the pickups) during the cooling cycle so that system gain would increase as the beam size decreased for a fixed amount of kicker power. The combined result of both components of the upgrade was to cool $80 \times 10^{6} 8 \mathrm{GeV}$ particles from $30 \pi$-mm-mrad to 5 $\pi$-mm-mrad in 1.5 seconds. This would have been sufficient for the anticipated Run II antiproton flux of $20 \times 10^{10}$ antiprotons/hour.

However, for stacking rates beyond $20 \times 10^{10}$ antiprotons/hour, the Debuncher cooling systems would near optimum cooling gain and further increases in

\footnotetext{
"Work supported by the United States Department of Energy under contract No. DE-AC02-76CH03000

"Email: mcginnis@fnal.gov
}

stacking rate would be limited by cooling bandwidth. [1]

Also, the 2-4 GHz system left little margin for design error. If the design plunging aspect ratio could not be achieved (due to factors such as closed orbit variations for example) or the front-end signal-to-noise ratio was smaller than anticipated (due to larger than expected combining losses or lack of pickup sensitivity), Run II stacking rates would not be achieved. To provide a future upgrade path and give larger design margins, the upgrade plan was changed to increase the Debuncher cooling bandwidth to $4-8 \mathrm{GHz}$.

\section{NARROW BAND SYSTEM DESIGN}

For Run $1 b$, all of the stochastic cooling pickup and kicker arrays at Fermilab consisted of stripline or planar loop electrodes. The signals from these electrodes are combined with a binary combiner tree formed by microstrip or stripline transmission lines. With a binary combining scheme, there must be no waveguide modes traveling down the beam pipe that would provide an alternate (uncontrollable) signal path in parallel to the binary combiner tree.

The nominal Debuncher transverse aperture is $30 \pi$ $\mathrm{mm}$-mrad (95\% un-normalized). To account for closedorbit variations, the design aperture of the cooling arrays was set at $40 \pi-\mathrm{mm}$-mrad. With lattice beta functions on the order of 10 meters, the transverse dimensions of the beam pipe will be about $40 \mathrm{~mm}$ which will propagate waveguide modes above $4 \mathrm{GHz}$. The presence of travelling waveguide modes in the beam pipe will limit the workable fractional bandwidth of the cooling arrays.

The solution was to divide the $4-8 \mathrm{GHz}$ bandwidth into 4 narrower bands with each band having a bandwidth of about $1 \mathrm{GHz}$. The cooling arrays are built with slot coupled "slow-wave" waveguide structures as shown in Figure 1. The structure consists of two rectangular waveguides that are coupled to a rectangular beam pipe by a series of slots. The transverse signal is derived from the difference between the two waveguides and the momentum signal is derived from the sum of the two waveguides.

The image current that flows along the walls of the beam pipe due to a charged particle beam travelling in the center of the beam pipe excites electromagnetic magnetic waves in the slots which in turn excite travelling waveguide modes in the side waveguides and beampipe. Since the phase velocity of the unperturbed waveguide modes is faster than the beam velocity, the slots also act to "slow down" the waveguide modes by multiple reflections so that the phase velocity of the waveguide modes along the structure matches the beam 
velocity. These structures are similar to the ridged waveguide pickups used for bunched beam cooling in the CERN SPS.[2]

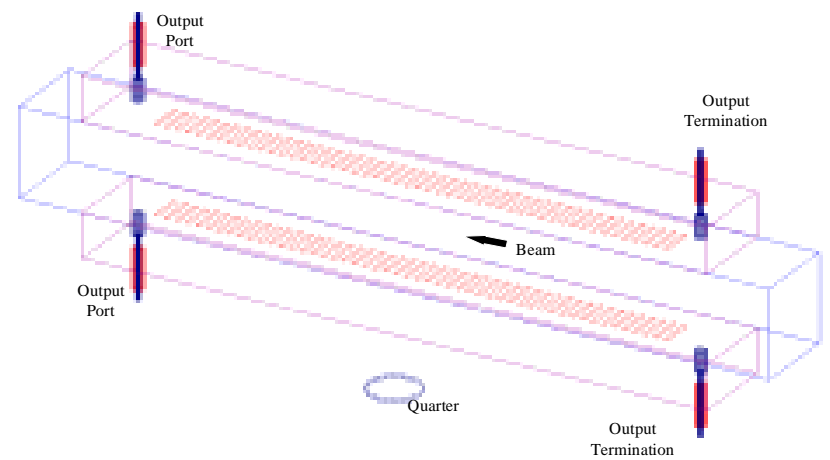

Figure 1: Conceptual drawing of a slow wave pickup.

The gain of the structure is proportional to the number of waveguide slots or the length of the array. However, the bandwidth of the structure is inversely proportional to the length of the array. For a $40 \pi$-mm-mrad aperture in the $4-8 \mathrm{GHz}$ range, a 0.7 -meter long array provides about $1 \mathrm{GHz}$ of bandwidth. In the Debuncher ring, there is 11.4 meters of space available per plane for either pickup or kicker, which leaves enough room for 16 arrays per plane (or 4 arrays per band). Because the fractional bandwidth of each band is still relatively large, it is desirable from a perspective of power balance to power each kicker array with one power amplifier. Because of the large fractional bandwidth per band and the frequency range of $4-8 \mathrm{GHz}$, the natural choice for power amplifier is a travelling wave tube (TWT). The TWTs chosen can provide 150 Watts per tube, which results in an available power of 2400 Watts per plane. The Run II design goals for a $4-8 \mathrm{GHz}$ system require about 1200 Watts per plane, which leaves plenty of room for future upgrades or design margin. [1]

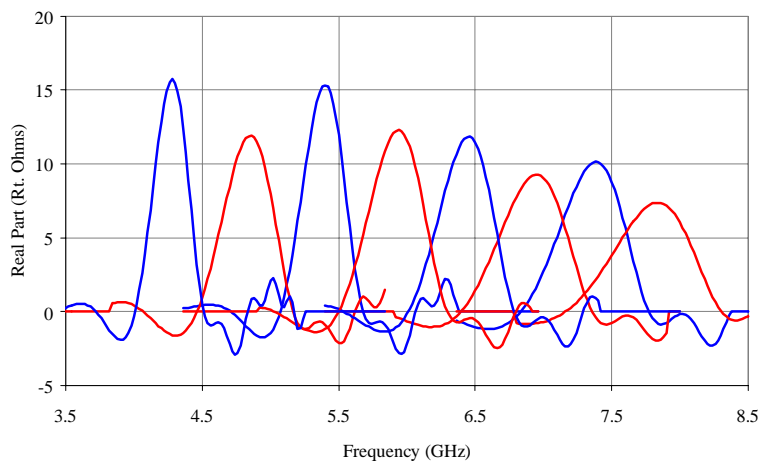

Figure 2: Pickup impedance for all 8 sub-bands. The blue trace is the lower sub-band. The red trace is the upper sub-band.

The pickup arrays will be cooled with liquid helium to a temperature of about $10 \mathrm{~K}$. Before the pickup signals can be brought out to room temperature, they must be amplified with low noise cryogenic amplifiers, which are also cooled with liquid helium. The noise temperature of these amplifiers is about $25 \mathrm{~K}$, which brings the total front-end noise temperature to $35 \mathrm{~K}$.

Figure 3: The kicker response for all 4 bands. The green

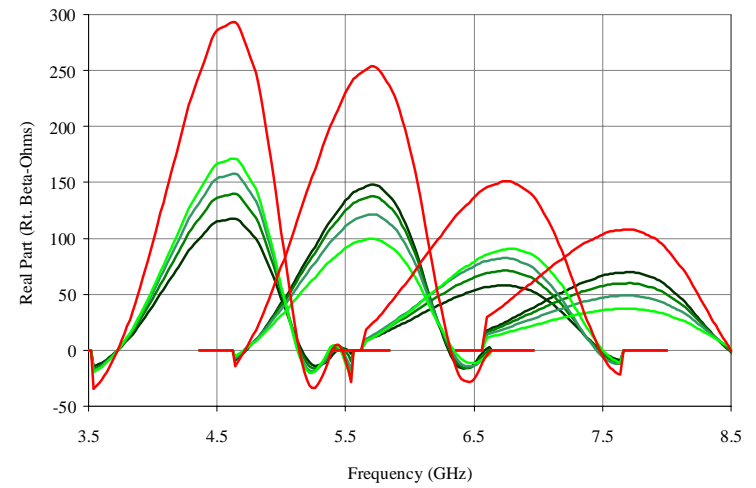

traces are the response of individual arrays while the red traces are the power sums of all the individual arrays in a band.

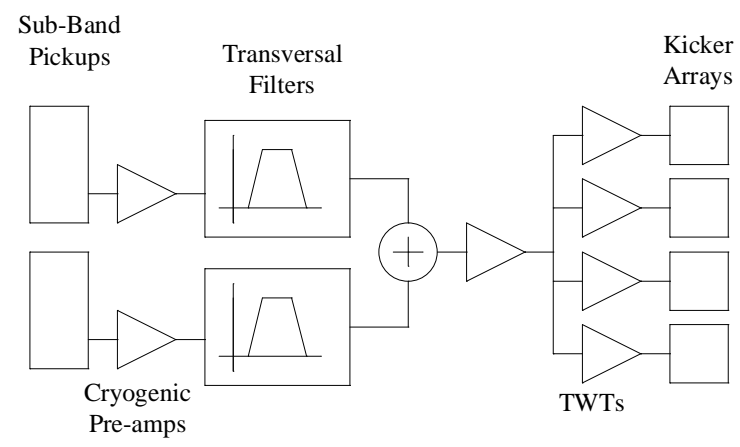

Figure 4: Simple schematic of components in a single band.

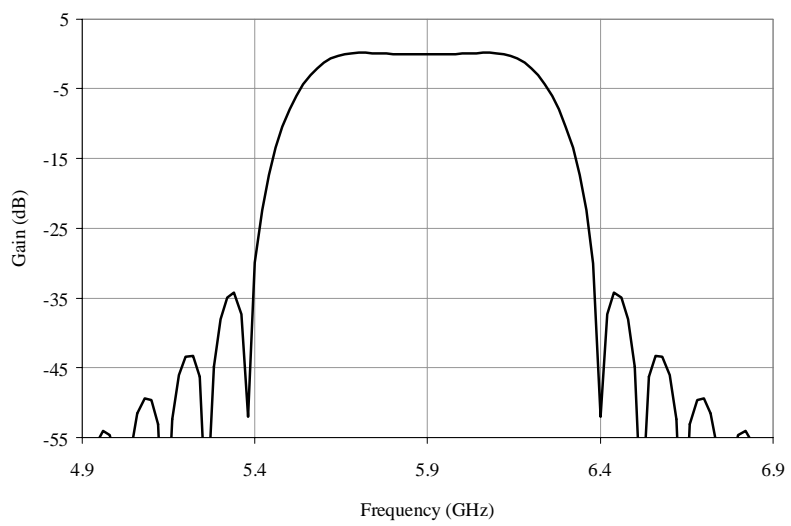

Figure 5: Response of a transversal filter for Band $2 b$.

If the pickup arrays have the same bandwidth as the kicker arrays, this would require eight cryogenic amplifiers per plane per band ( 4 for transverse, 4 for momentum). For reliability reasons, it is undesirable to have this many cryogenic amplifiers in the same vacuum vessel. The number of cryogenic amplifiers per band per 
plane could be reduced by adding a combiner board inside the cryogenic vacuum tank that adds the signals of a number arrays together. However, this combiner board would add extra loss to the pickup array resulting in a reduction in signal to noise ratio. Also the combiner board would be relatively large and make the cryogenic vacuum tank much more costly and difficult to assemble and repair.

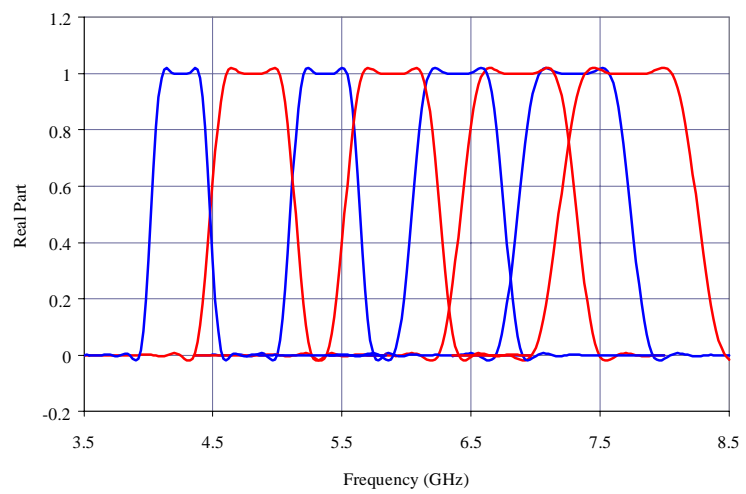

Figure 6: Transversal filter response for all 8 sub-bands. The blue trace is the lower sub-band. The red trace is the upper sub-band.

The alternative that was pursued was to divide each 1 $\mathrm{GHz}$ band into two $0.5 \mathrm{GHz}$ sub-bands with a center to center spacing of about $0.5 \mathrm{GHz}$. The length of the pickup arrays was increased to 1.4 meters so that there was only one pickup array per sub-band per plane. This resulted in four cryogenic amplifiers per band per plane (1 transverse amplifier $\times 2$ sub-bands per band +1 momentum amplifier $\times 2$ sub-bands per band) and no cryogenic combiner boards.

The transverse impedance of the pickup array is defined as:

$$
\mathrm{P}_{\Delta}=\frac{1}{2}\left(\mathrm{Z}_{\Delta}\right) \mathrm{i}_{\mathrm{b}}{ }^{2} \frac{\varepsilon_{\mathrm{b}}}{1 \pi-\mathrm{mm}-\operatorname{mrad}}
$$

Where $\mathrm{P}_{\Delta}$ is the signal power obtained from the pickup, $i_{b}$ is the beam current and $\varepsilon_{b}$ is the emittance of the beam. The kicker impedance is defined as:

$$
\mathrm{P}_{\Delta}=\frac{1}{2} \frac{\left(\sqrt{\beta_{\mathrm{k}}} \frac{\Delta \mathrm{pc}}{\mathrm{q}}\right)^{2}}{\mathrm{Z} \beta_{\Delta \mathrm{kr}}}
$$

Where $\Delta \mathrm{pc}$ the change in transverse momentum going through the kicker for a particle with charge $\mathrm{q}$ and $\beta_{\Delta_{\mathrm{kr}}}$ is the lattice beta function at the kicker. The array impedance is defined in this manor so as to incorporate the lattice beta functions easily into cooling rate calculations. A plot of the pickup impedance for all 4 bands is show in Figure 2. A plot of the kicker impedance for all 4 bands is show in Figure 3.

The two sub-bands for each band were added together as shown in Figure 4. Because the noise power spectral density of the cryogenic amplifier and pickup array covered a frequency range much wider than the bandwidth of the pickup, a narrow-band filter has to be added to each sub-band before the sub-bands are added together.

This narrow-band filter has to have "brick-wall" characteristics of flat phase and gain in the filter passband with very high rejection outside the pass-band. These characteristics can be accomplished with a transversal filter made with many sections of weakly coupled striplines. The response of such a filter is shown in Figure 5. The responses of all the filters in the 4-8 $\mathrm{GHz}$ band are shown in Figure 6.

The total system impedance for all 4 bands is shown in Figure 7. The predicted transfer efficiency from the Debuncher to the Accumulator for Run II intensities is using the impedance profile shown in Figure 7 is shown in Figure 8.

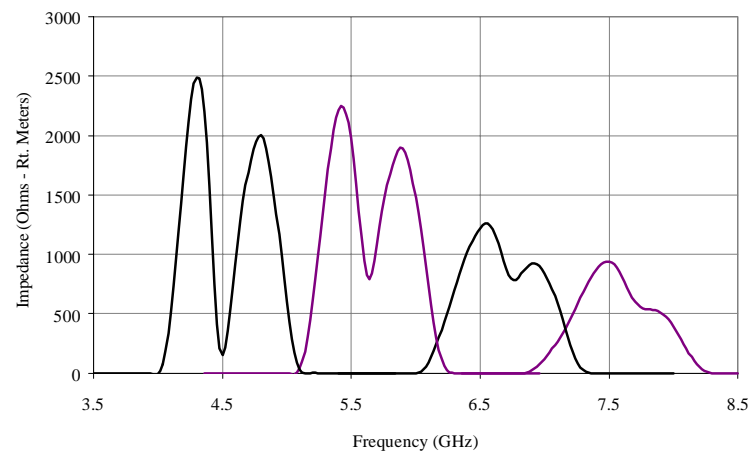

Figure 7: The total system impedance for all 4 bands. Bands I and III are the black traces. Bands II and IV are the purple traces.

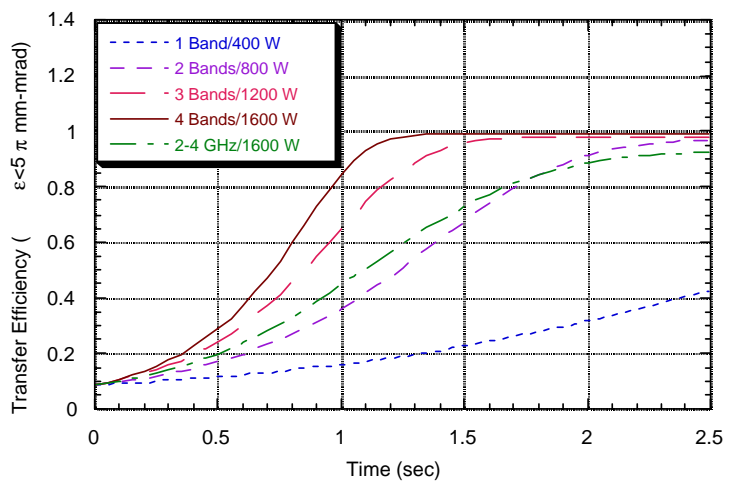

Figure 8: Debuncher to Accumulator transfer efficiency for Run II parameters using the 4-8 GHz narrow band system.

\section{REFERENCES}

[1] J. Marriner,"Debuncher Stochastic Cooling Upgrade for Run II and Beyond", Fermilab PBAR Note 573, April, 1998.

[2] D. Boussard, G. Di Massa, "High Frequency Slow Wave Pickups",CERN SPS/86-4, February 1986 